\title{
Estudo da sinterização da zircônia dopada com óxidos de terras raras a 5 GPa de pressão
}

\section{(Sintering of rare earth-doped zirconia under 5 GPa pressure)}

\author{
C. Kuranagal, F. S. de Azevedo Ribeiro' ${ }^{2}$, M. Filgueira ${ }^{2}$ \\ ${ }^{1}$ Laboratório Associado de Sensores e Materiais - LAS, \\ Centro de Tecnologias Especiais - CTE, Instituto Nacional de Pesquisas Espaciais - INPE \\ Av. dos Astronautas, 1758, Jd. da Granja, S. José dos Campos, SP 12245-970. \\ ${ }^{2}$ Laboratório de Materiais Avançados - LAMAV, Centro de Ciência e Tecnologia - CCT \\ Universidade Estadual do Norte Fluminense - UENF \\ Av. Alberto Lamego, 2000, Pq. Califórnia, Campos dos Goytacazes, RJ 28013-600 \\ kuranaga@las.inpe.br,marcello@uenf.br
}

\begin{abstract}
Resumo
A zircônia $\left(\mathrm{ZrO}_{2}\right)$ tem mostrado grande destaque entre as cerâmicas avançadas, atraindo muito o interesse de pesquisadores em seus vários campos de atuação. A zircônia apresenta elevada resistência quando na fase tetragonal, mas a fase estável a temperatura ambiente é a monoclínica, sendo necessário o uso de estabilizantes para a fase tetragonal. Neste trabalho propomos a sinterização rápida da zircônia parcialmente estabilizada com óxidos de terras raras ( $\left.\mathrm{ZrO}_{2}-\mathrm{OTR}\right)$, mediante o emprego da alta pressão de 5 GPa. As condições de sinterização realizadas neste trabalho são inovadoras, haja visto que utilizou-se de tecnologia alternativa para processar a $\mathrm{ZrO}_{2}$ OTR, chamada de altas temperaturas e altas pressões (HPHT). Foi utilizada uma pressão de $5 \mathrm{GPa}$, temperaturas de 1100,1200 e $1300{ }^{\circ} \mathrm{C}$ nos tempos de 2 e $5 \mathrm{~min}$. O melhor resultado foi obtido nas amostras sinterizadas a $5 \mathrm{GPa} / 1300{ }^{\circ} \mathrm{C} / 5 \mathrm{~min}$, onde apresentaram microdureza média de $488,73 \mathrm{kgf} / \mathrm{mm}^{2}$, para uma tenacidade à fratura de 5,33 MPa.m², as quais apresentaram densidade da ordem de $97,88 \%$ da teórica, e $88 \%$ em volume de fase tetragonal retida à temperatura ambiente.
\end{abstract}

Palavras-chave: $\mathrm{ZrO}_{2}$, óxidos de terras raras, sinterização, altas pressões, fase tetragonal.

Abstract

Zirconia $\left(\mathrm{ZrO}_{2}\right)$ has shown great projection among the advanced ceramics, attracting the interest of researchers in its various fields of application. Tetragonal zirconia presents high mechanical strength, but the room temperature stable phase is the monoclinic, being necessary the use of stabilizers for obtaining the tetragonal phase. In this work the rapid sintering of zirconia partially stabilized with rare earth oxides ( $\left.\mathrm{ZrO}_{2}-\mathrm{OTR}\right)$, via $5 \mathrm{GPa}$ high pressure is proposed. The sintering conditions employed in this work are innovative, due to the use of an alternative technology to process $\mathrm{ZrO}_{2}$-OTR, so called high temperature - high pressure (HPHT). A pressure of 5 GPa and temperatures of 1100,1200 and $1300^{\circ} \mathrm{C}$ for times of 2 and 5 min were used. The best results were obtained for samples sintered at 5 GPa at $1300^{\circ} \mathrm{C} / 5 \mathrm{~min}$., where a micro-hardness of $488,73 \mathrm{kgf} / \mathrm{mm}^{2}$, a fracture toughness of 5,33 MPa.m ${ }^{1 / 2}$, a density of $97,88 \%$, and a $88 \mathrm{vol} . \%$ of tetragonal phase retained at room temperature were achieved.

Keywords: $\mathrm{ZrO}_{2}$, rare earth oxides, sintering, high pressures, tetragonal phase.

\section{INTRODUÇÃO}

A zircônia $\left(\mathrm{ZrO}_{2}\right)$ tem mostrado grande destaque entre as cerâmicas avançadas, atraindo muito interesse de pesquisadores em seus vários campos de atuação. As aplicações mais promissoras são como cerâmicas estruturais (partes de motores de combustão, palhetas de turbinas, ferramentas de corte, partes de implantes ortopédicos) e como eletrólitos sólidos (sensores de oxigênio, células de combustível, bombas de oxigênio) [1].

A zircônia pura apresenta três fases cristalinas, à pressão ambiente, que são: monoclínica - $\mathrm{m}$ (até $1170{ }^{\circ} \mathrm{C}$ ), tetragonal - t (de 1170 até $\left.2370{ }^{\circ} \mathrm{C}\right)$, e cúbica - c (de 2370 a $\left.2680{ }^{\circ} \mathrm{C}\right)$ [2]. Devido à elevada variação volumétrica associada à transição t-m, a zircônia pura não apresenta aplicabilidade prática como material de engenharia. A adição de certos óxidos estabilizantes é imprescindível para manter as fases polimórficas de temperaturas elevadas, em temperatura ambiente. Dentre estes aditivos, a ítria $\left(\mathrm{Y}_{2} \mathrm{O}_{3}\right)$ se destaca como o estabilizante mais utilizado para a estabilização de fase tetragonal do $\mathrm{ZrO}_{2}$, o qual é conhecido como fase tenaz e dura, à temperatura ambiente, o que possibilita o uso deste material como cerâmica avançada [3], e notadamente em aplicações como ferramenta de corte.

Aestabilidade dos polimorfos do $\mathrm{ZrO}_{2}$ depende fortemente do tipo e quantidade de óxido que é usado para diminuir a temperatura da transformação t-m. Feder et al. [4] e Piconi e Maccauro [5] afirmam que trata-se de uma transformação de natureza martensítica, a qual é acompanhada por um aumento volumétrico $(\Delta \mathrm{V})$ da ordem de $4 \%$. Normalmente 
quando tal transformação se torna ativa, é gerado um campo de tensões nas proximidades das trincas, geradas devido a $\Delta \mathrm{V}$, o que se traduz num aumento da tenacidade à fratura do material $\left(\mathrm{K}_{\mathrm{Ic}}\right)$.

Em sua pesquisa, Kuranaga [6] e mais recentemente Nono e Freitas [7], mostraram que é uma forte alternativa o uso da mistura de óxidos de ítrio e de terras raras (OTR), em substituição à $\mathrm{Y}_{2} \mathrm{O}_{3}$ pura, como agente estabilizante da fase tetragonal do $\mathrm{ZrO}_{2}$ à temperatura ambiente, resultando em redução de custos de processamento dos pós, e manutenção das propriedades mecânicas de interesse. Para tal, foi utilizada a rota da co-precipitação para a obtenção dos pós de $\mathrm{ZrO}_{2}$-OTR, onde os estudos mostraram que a obtenção da zircônia totalmente tetragonal é possível, mediante adição de $6 \%$ em peso de OTR. Kuranaga [6] mostrou que melhores propriedades mecânicas são obtidas quando o material é submetido à sinterização a temperaturas superiores a $1400{ }^{\circ} \mathrm{C}$, para tempos de $3 \mathrm{~h}$.

Durante a década de 60 e 70 alguns pesquisadores, dos quais se destacam Weber [8], Vahldiek et al. [9], Bendeliani et al. [10], e Liu [11], e mais recentemente, no final dos anos 90, Haines et al. [12] dedicaram esforços no sentido de estudar o comportamento do polimorfismo da $\mathrm{ZrO}_{2}$ via altas pressões. Vale salientar que os mesmos estavam interessados apenas na fase ortorrômbica, para fins científicos, a qual só foi encontrada via uso de altas pressões e altas temperaturas (HPHT), conforme pode ser observado no gráfico da Fig.1, retirado de Liu [11].

Neste contexto, o presente trabalho visa a obtenção de corpos densos à base de $\mathrm{ZrO}_{2}-6 \%$ peso OTR, via rota alternativa de processamento, a qual emprega altas temperaturas, altas pressões, e tempos curtos de sinterização (HPHT), e caracterização da micro-dureza e tenacidade à fratura das amostras sinterizadas, aliada à determinação das fases presentes. Cabe ressaltar que o trabalho visa à obtenção da fase tetragonal como majoritária, para averiguação da

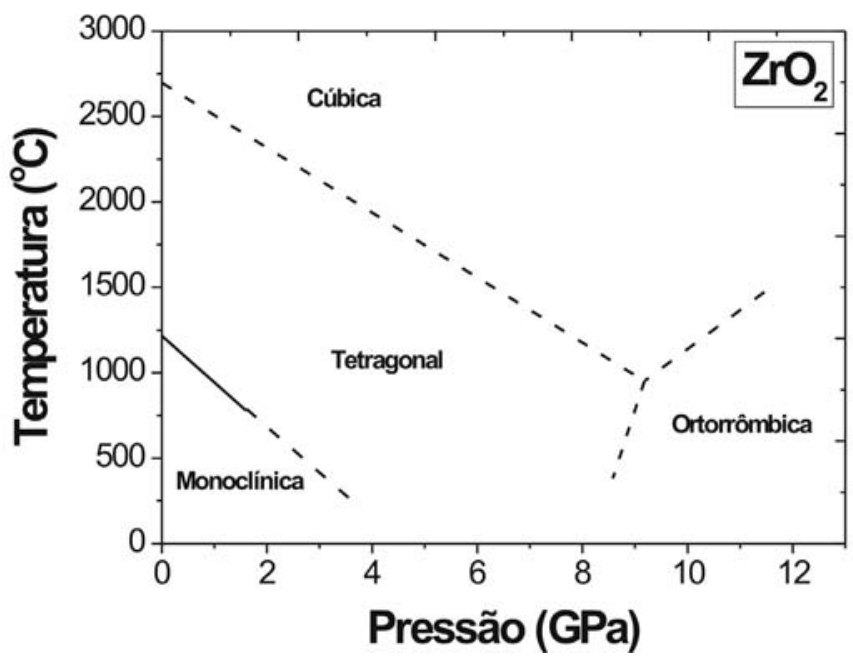

Figura 1: Diagrama P-T de fases hipotético da zircônia. [Figure 1: P-T phase diagram for zirconia.] possibilidade do uso da tecnologia HPHT na confecção de ferramentas de corte à base de zircônia.

\section{METODOLOGIA}

A rota de obtenção dos pós de $\mathrm{ZrO}_{2}-6 \%$ peso OTR é bem descrita por Kuranaga [6] e por Nono e Freitas [7], nos quais os materiais de partida foram o oxicloreto de zircônio octahidratado $\left(\mathrm{ZrOCl}_{2}\right)$, e o concentrado de óxidos de terras raras (OTR). Os pós foram obtidos via co-precipitação química, usando hidróxido de amônia $\left(\mathrm{NH}_{4} \mathrm{OH}\right)$ como agente precipitante. $\mathrm{O}$ pó resultante co-precipitado foi secado em estufa e calcinado a $400{ }^{\circ} \mathrm{C}$, e depois desaglomerado. O tamanho médio de partícula foi de 1,25 $\mu \mathrm{m}$. Abaixo é mostrada a Tabela I com a análise química da fração OTR, onde se pode observar que a mesma apresenta $44,56 \%$ de ítria em sua composição. A Tabela II fornece as condições de sinterização para os grupos de amostras.

Tabela I - Análise química da mistura de óxidos de ítrio e de terras raras (OTR).

[Table I - Chemical analysis of the mixture of yttrium and rare earth oxides (OTR).]

\begin{tabular}{cccc}
\hline Elementos & $\%$ (em massa) & Elementos & $\%$ (em massa) \\
\hline $\mathrm{Y}_{2} \mathrm{O}_{3}$ & 44,56 & $\mathrm{Gd}_{2} \mathrm{O}_{3}$ & 1,18 \\
$\mathrm{Yb}_{2} \mathrm{O}_{3}$ & 19,98 & $\mathrm{~Tb}_{4} \mathrm{O}_{7}$ & 0,77 \\
$\mathrm{Er}_{2} \mathrm{O}_{3}$ & 13,80 & $\mathrm{Sm}_{2} \mathrm{O}_{3}$ & 0,39 \\
$\mathrm{Dy}_{2} \mathrm{O}_{3}$ & 10,17 & $\mathrm{Nd}_{2} \mathrm{O}_{3}$ & 0,17 \\
$\mathrm{Ho}_{2} \mathrm{O}_{3}$ & 3,06 & $\mathrm{CeO}_{2}$ & 0,09 \\
$\mathrm{Tm}_{2} \mathrm{O}_{3}$ & 2,76 & $\mathrm{ThO}_{2}$ & 0,05 \\
$\mathrm{Lu}_{2} \mathrm{O}_{3}$ & 2,70 & $\mathrm{Eu}_{2} \mathrm{O}_{3}$ & 0,02 \\
\hline
\end{tabular}

Tabela II - Condições de sinterização das amostras de $\mathrm{ZrO}_{2}-6 \%$ peso OTR, tratadas a $5 \mathrm{GPa}$. Foram sinterizadas 5 amostras em cada condição.

[Table II - Sintering conditions for the $\mathrm{ZrO}_{2}-6 \%$ wt OTR samples, treated at 5 GPa. 5 samples were sintered in each condition.]

\begin{tabular}{ccc}
\hline Amostra & $\mathrm{T}\left({ }^{\circ} \mathrm{C}\right)$ & $\mathrm{t}(\min )$. \\
\hline $\mathrm{A}$ & 1100 & 2 \\
$\mathrm{~B}$ & 1100 & 5 \\
$\mathrm{C}$ & 1200 & 2 \\
$\mathrm{D}$ & 1200 & 5 \\
$\mathrm{E}$ & 1300 & 2 \\
$\mathrm{~F}$ & 1300 & 5 \\
\hline
\end{tabular}

O pó foi pré-compactado em prensagem uniaxial em $125 \mathrm{MPa}$ (prensa DAN-PRESSE). Foi utilizada uma massa de pó de $\mathrm{ZrO}_{2}-6 \%$ peso OTR de 0,425 g por amostra. Em seguida, os corpos verdes foram submetidos aos tratamentos termobáricos, os quais foram realizados em prensa hidráulica especial de 2500 ton, da Ryazantyashpressmash (Rússia). 
Cada amostra foi submetida à pressão de trabalho, $5 \mathrm{GPa}$, e após atingir este valor, o sistema elétrico de aquecimento direto, via transformador, é acionado e a corrente passa pela amostra, promovendo o aquecimento da mesma; assim, chega-se à temperatura desejada em poucos segundos. Com o fim do tratamento, a energia é desligada, e o sistema resfria naturalmente, e após cerca de 1 min, a pressão é lentamente aliviada no sistema. Maiores detalhes sobre o procedimento de sinterização de materiais via HPHT, vide ref.13.

As densidades dos sinterizados foram calculadas segundo o método de Arquimedes, em uma balança Scaltec SBC 31 de $0,0001 \mathrm{~g}$ de resolução. Testes de micro-dureza Vickers foram realizadas no dispositivo de microdureza acoplado a um microscópio metalográfico Zeiss, modelo Jenavert. Foi utilizada uma carga de $50 \mathrm{kgf}$ durante $15 \mathrm{~s}$. Foram realizadas 5 repetições em cada amostra em forma de cruz. Os valores de microdureza foram calculados pela expressão A [14]:

$$
H v=\frac{1854 \times C}{d^{2}}
$$

$\mathrm{Hv}=$ microdureza Vickers $\left(\mathrm{Kgf} / \mathrm{mm}^{2}\right), \mathrm{C}=$ carga aplicada $(\mathrm{Kgf})$ e d $=2 \mathrm{a}=$ diagonal média $(\mathrm{mm})$.

A tenacidade à fratura das amostras foi determinada via equação B [15], por se tratar da equação mais adequada para este material.

$K_{I C}=0,0363 \cdot\left(\frac{E}{H_{V}}\right)^{2 / 5} \cdot\left(\frac{P}{a^{1,5}}\right) \cdot\left(\frac{a}{c}\right)^{1,56}$

$\mathrm{K}_{\mathrm{IC}}=$ tenacidade à fratura $\left(\mathrm{MPa} \cdot \mathrm{m}^{1 / 2}\right), \mathrm{E}=$ módulo elástico (MPa), $\mathrm{H}=$ micro-dureza Vickers $(\mathrm{MPa}), \quad \mathrm{P}=$ carga da indentação $(\mathrm{N}), \mathrm{a}=$ metade da diagonal da indentação $(\mathrm{m}), \mathrm{e}$ $\mathrm{c}=$ metade do comprimento médio da trinca $(\mathrm{m})$.

Para a análise das fases nas amostras sinterizadas (pastilhas), foi utilizado um difratômetro de raios X, Seifert URD 65, empregando radiação $\mathrm{CuK} \alpha$, a um passo de $0,02^{\circ}$ e tempo de $1 \mathrm{~s}$. Os resultados de difração também foram utilizados para o cálculo das frações das fases tetragonal e monoclínica de cada amostra, segundo a equação C [16].

$$
X_{m}=\frac{I_{m}(111)+I_{m}(11 \overline{1})}{I_{m}(111)+I_{m}(11 \overline{1})+I_{t}(111)}
$$

$\mathrm{X}_{\mathrm{m}}$ = fração da fase monoclínica, $\mathrm{I}_{\mathrm{m}}$ e $\mathrm{I}_{\mathrm{t}}$ correspondem, respectivamente, às intensidades dos picos de fases monoclínica e tetragonal, os índices de Miller, (hkl), significam os planos considerados nas medidas de intensidade.

\section{RESULTADOS E DISCUSSÃO}

De acordo com a Fig.1, e com as condições de processamento mostradas na Tabela II, todas as amostras foram sinterizadas na região de estabilidade da fase tetragonal. As amostras A apresentaram elevada fragilidade, o que impossibilita as suas caracterizações. Isto é atribuído ao fato da presença de grande quantidade de trincas nestas amostras, o que é característico da transformação t-m. De acordo com a Fig. 1, seria possível a estabilização total da fase tetragonal da zircônia a temperaturas inferiores a $1000{ }^{\circ} \mathrm{C}$, para pressões da ordem de $2 \mathrm{GPa}$, indicando que todas as condições de sinterização utilizadas neste trabalho deveriam, a princípio, promover o surgimento de elevadas quantidades de fase tetragonal nas amostras sinterizadas. Isto não ocorre, conforme veremos adiante, face à instabilidade termodinâmica da região de sinterização HPHT deste trabalho, onde observam-se linhas de incerteza.

A Fig. 2 mostra os difratogramas de raios $\mathrm{X}$ das amostras $\mathrm{B}$ a F. Pode-se claramente observar que há uma similaridade entre todos os difractogramas, apesar da amostra $\mathrm{F}$ apresentar, sensivelmente, maior quantidade e intensidade de picos relativos à fase tetragonal.

Em concordância com os resultados de difração de raios $X$, e via uso da equação $C$, foi feito o gráfico da Fig. 3, que fornece os percentuais de fases me t para cada amostra. Podese observar que o percentual da fase $t$ aumenta, à medida em que se eleva a temperatura e/ou tempo de sinterização, para a pressão de $5 \mathrm{GPa}$. Isto é explicado pelo fato de que a transição $\mathrm{t}-\mathrm{m}$ ocorre a $1170{ }^{\circ} \mathrm{C}$ (vide ref.2), à pressão atmosférica. Desta forma, o uso de temperaturas inferiores de sinterização favorece o surgimento desta fase $(\mathrm{m})$. Também deve ser notado que a linha de separação entre as fases $m$ e t não está totalmente no equilíbrio termodinâmico, havendo uma zona de incerteza. Isto indica que para as condições de sinterização das amostras B, a pressão de 5 GPa não se apresenta como parâmetro de influência na manutenção da fase $\mathrm{t}$, na temperatura de $1100{ }^{\circ} \mathrm{C}$. As amostras C e D

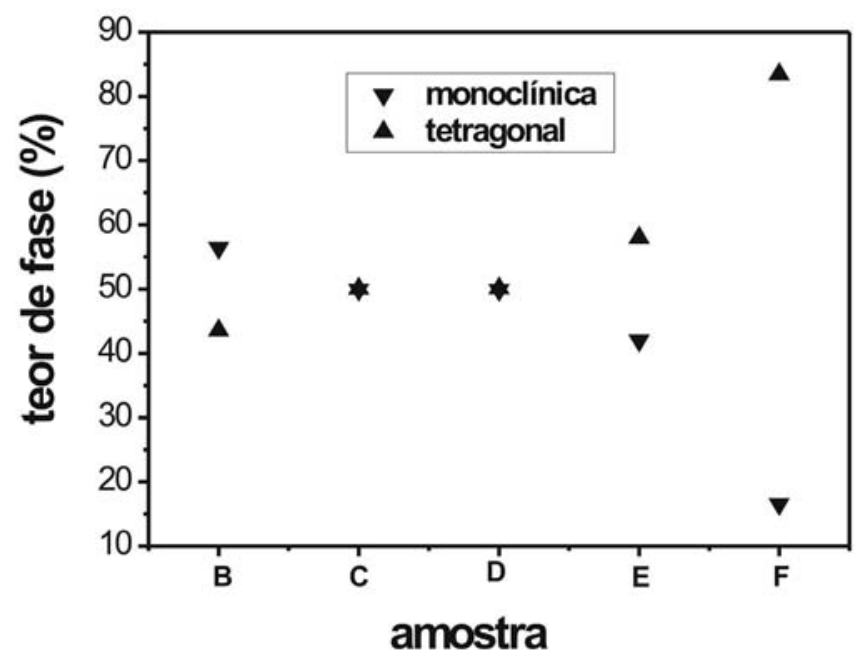

Figura 2: Difratogramas de raios $\mathrm{X}$ das amostras sinterizadas sob pressão.

[Figure 2: X-ray diffraction patterns of the high pressure sintered samples.] 


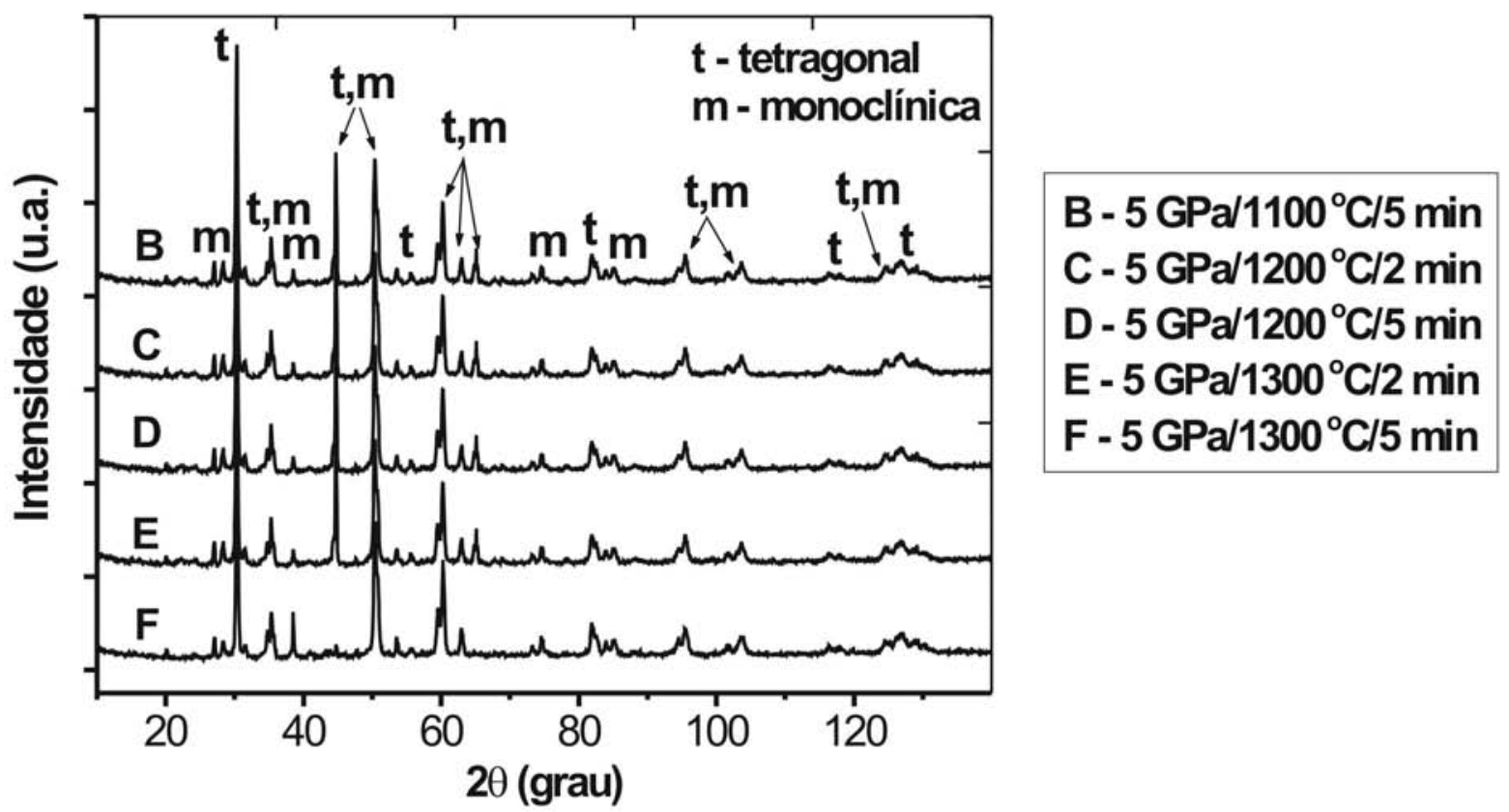

Figura 3: Percentuais de fases monoclínica e tetragonal para cada amostra. [Figure 3: Monoclinic and tetragonal phase content for each sample.]

apresentam o mesmo percentual de fases $\mathrm{t}$ e $\mathrm{m}$, indicando que para temperaturas da mesma ordem em que ocorre a transição t-m, no caso a $1200{ }^{\circ} \mathrm{C}$, o incremento no tempo de sinterização é inexpressivo. Com o aumento da temperatura até $1300{ }^{\circ} \mathrm{C}$, pode-se observar um aumento de fase t. As amostras E apresentam $60 \%$ em volume de fase t. Já as amostras $\mathrm{F}$ apresentam $88 \%$ em volume de fase $\mathrm{t}$, indicando que para temperaturas superiores à da transição $\mathrm{t}-\mathrm{m}$, o tempo de sinterização torna-se um fator bastante influente, para a pressão de $5 \mathrm{GPa}$.

A Tabela III fornece os resultados de densificação, microdureza, e tenacidade à fratura das amostras. Pode-se observar uma tendência crescente da densidade das amostras mediante o emprego de temperaturas e/ou tempos mais elevados de sinterização. As amostras F apresentam, então, os valores mais elevados de densidade. Estes resultados estão em pleno acordo com a expansão volumétrica verificada anteriormente

Tabela III - Correlações entre densidade, micro-dureza e tenacidade à fratura das amostras.

[Table III - Correlations among density, micro-hardness and fracture toughness of the samples.]

\begin{tabular}{|c|c|c|c|}
\hline amostra & $\begin{array}{l}\text { Densidade } \\
\text { relativa (\%) }\end{array}$ & $\begin{array}{c}\text { Micro-dureza,Hv } \\
\left(\mathrm{kgf} / \mathrm{mm}^{2}\right)\end{array}$ & $\begin{array}{c}\mathrm{K}_{\mathrm{Ic}} \\
\left(\mathrm{MPa} \cdot \mathrm{m}^{-1 / 2}\right)\end{array}$ \\
\hline B & $92,85 \pm 0,31$ & $369,33 \pm 10,53$ & $4,32 \pm 0,31$ \\
\hline $\mathrm{C}$ & $93,97 \pm 0,88$ & $389,12 \pm 17,32$ & $4,73 \pm 0,83$ \\
\hline $\mathrm{D}$ & $94,73 \pm 0,57$ & $408,62 \pm 11,71$ & $4,64 \pm 0,48$ \\
\hline $\mathrm{E}$ & $96,56 \pm 0,32$ & $450,54 \pm 11,38$ & $4,81 \pm 0,43$ \\
\hline $\mathrm{F}$ & $97,88 \pm 0,29$ & $488,73 \pm 15,67$ & $5,33 \pm 0,59$ \\
\hline
\end{tabular}

$[4,5]$, visto que a transformação $\mathrm{t}-\mathrm{m}$ reduz sensivelmente a densidade dos sinterizados de $\mathrm{ZrO}_{2}$. Os resultados de microdureza podem ser explicados segundo o mesmo raciocínio acima descrito, visto que a fase $t$ apresenta dureza superior à fase m. Esta expansão volumétrica promovida pela transição t-m pode ser observada, sobretudo nas amostras que apresentam maior percentual de fase m (amostras A a D).

Com relação aos resultados de $\mathrm{K}_{\mathrm{Ic}}$, observa-se o sensível aumento neste parâmetro com a retenção da fase t. Contudo, para as amostras C e D, nota-se que os resultados estão na mesma ordem de grandeza, via análise dos desvios-padrão obtidos. Isto pode ser explicado pelo fato destas amostras apresentarem o mesmo percentual de fase $t$ (vide Fig. 3). As amostras $\mathrm{F}$ apresentam $\mathrm{K}_{\mathrm{Ic}}=5,33 \mathrm{MPa} \cdot \mathrm{m}^{1 / 2}$, o mais alto valor obtido para as amostras estudadas, em face do elevado teor de fase $t$.

Diante destes resultados, observa-se que o uso de alta pressão apresenta influência marcante na obtenção de amostras densas de $\mathrm{ZrO}_{2}$ na fase tetragonal, em tempos muito curtos de sinterização. Mesmo assim, a amostra F apresenta $12 \%$ em volume de fase monoclínica, o que pode ser atribuído ao brusco resfriamento pós-sinterização, retendo esta quantia de fase monoclínica, a qual foi formada durante o início da sinterização. Vale, ainda, informar que Nono et al. [1], utilizando o mesmo material deste trabalho, alcançaram o valor máximo de $\mathrm{K}_{\mathrm{Ic}}=4,3 \mathrm{MPa} \cdot \mathrm{m}^{1 / 2}$, para amostras sinterizadas convencionalmente a $1400{ }^{\circ} \mathrm{C} / 3 \mathrm{~h}$, mesmo atingindo o mesmo nível de densificação das amostras $\mathrm{F}$ deste trabalho. Isto é um indicativo que o uso de altas pressões pode ser uma rota tecnicamente viável para o processamento de ferramentas de corte à base de $\mathrm{ZrO}_{2}$. 


\section{CONCLUSÕES}

Neste trabalho exploratório, cujo alvo principal foi a obtenção de amostras densas de $\mathrm{ZrO}_{2}-6 \%$ peso OTR, via sinterização assistida por alta pressão, com fração tetragonal elevada, chegou-se aos seguintes pontos que merecem destaque:

- Para a pressão de $5 \mathrm{GPa}$, maiores teores de fase $\mathrm{t}$ foram obtidos via emprego de tempos e/ou temperaturas maiores de sinterização;

- Para temperaturas de sinterização abaixo da temperatura $\mathrm{t}-\mathrm{m}\left(1170{ }^{\circ} \mathrm{C}\right)$, a pressão não exerce qualquer influência;

- A alta pressão permitiu obter amostras densas e com elevados valores de fase tetragonal, mesmo para tempos muito curtos de sinterização;

- Os melhores resultados de densificação, micro-dureza, e tenacidade à fratura, respectivamente de 97,88\%, 488,73 $\mathrm{kgf} / \mathrm{mm}^{2}$, e 5,33 MPa.m ${ }^{1 / 2}$, foram obtidos para as amostras F, sinterizadas a $5 \mathrm{GPa} / 1300{ }^{\circ} \mathrm{C} / 5 \mathrm{~min}$, ou seja, para os maiores tempos e temperaturas de tratamento;

- Os resultados de $\mathrm{K}_{\mathrm{Ic}}$ obtidos neste trabalho para a amostra $\mathrm{F}$ foi superior ao da literatura, para o mesmo material, porém sinterizado convencionalmente.

\section{REFERÊNCIAS}

[1] M. C. A. Nono, Anais do Congresso Brasileiro de Engenharia e Ciência dos Materiais, S. Pedro, SP (2000) 18601.

[2] C. Kuranaga, M. C. A. Nono, Proc. of the Third
International Latin-American Conference on Powder Technology, Florianópolis, SC (2001) 661.

[3] X. Miao, D. Sun, P. W. Hoo, J. Liu, Y. Hu, Y. Chen, Ceram. Int., 30, 6 (2004) 1041.

[4] A. Feder, J. Alcala, L. Lianes, M. Anglada, J. Eur. Ceram. Soc. 23, 15 (2003) 2955.

[5] C. Piconi, G. Maccauro, Biomaterials 20, 1 (1999) 1.

[6] C. Kuranaga, "Preparação e caracterização de pós de $\mathrm{ZrO}_{2}$ obtidos a partir de uma zirconita brasileira", Diss. Mestrado, Faculdade de Engenharia Química de Lorena (1997).

[7] M. C. A. Nono, D. Freitas, Mater. Sci. Forum 416 (2003) 525 .

[8] E. C. Weber, J. Am. Ceram. Soc. 45 (1962) 614.

[9] F. W. Vahldiek, C. T. Lynch, L. B. Robinson, Science 142 (1963) 1059.

[10] N. A. Bendeliani, S. V. Popova, L. P. Verescha, Geochem. Int. 4, 3 (1967) 557.

[11] Liu, L-G. J. Phys. Chem. Solids, 41 (1979) 331.

[12] J. Haines, J. M. Leger, S. Hull, J. P. Petitet, J. Am. Ceram. Soc. 80, 7 (1997) 1910.

[13] G. S. Bobrovnitchii, W. S. Vianna, S. N. Monteiro, J. Superhard Mater. 133, 5 (2001) 9.

[14] M. F. Rodrigues, G. S. Bobrovnitchii, A. M. Ramalho M. Filgueira, Proceedings of the Fourth International LatinAmerican Conference on Powder Technology, Guarujá, SP (2003) 570.

[15] R. J. Anton, G. Subhash, Wear 239, 1 (2000) 27.

[16] T. Kosmac, R. Wagner, N. Claussen, J. Am. Ceram. Soc. 64, 4 (1981) C72.

(Rec. 18/09/04, Rev. 12/01/05, 16/04/05, Ac. 15/04/05) 